PROCEEDINGS OF THE

AMERICAN MATHEMATICAL SOCIETY

Volume 140, Number 6, June 2012, Pages 1921-1930

S 0002-9939(2011)11098-0

Article electronically published on October 5, 2011

\title{
ON THE FOURIER COEFFICIENTS OF 2-DIMENSIONAL VECTOR-VALUED MODULAR FORMS
}

\author{
GEOFFREY MASON
}

(Communicated by Kathrin Bringmann)

\begin{abstract}
Let $\rho: S L(2, \mathbb{Z}) \rightarrow G L(2, \mathbb{C})$ be an irreducible representation of the modular group such that $\rho(T)$ has finite order $N$. We study holomorphic vector-valued modular forms $F(\tau)$ of integral weight associated to $\rho$ which have rational Fourier coefficients. (These span the complex space of all integral weight vector-valued modular forms associated to $\rho$.) As a special case of the main theorem, we prove that if $N$ does not divide 120, then every nonzero $F(\tau)$ has Fourier coefficients with unbounded denominators.
\end{abstract}

\section{INTRODUCTION}

Let $\Gamma=S L(2, \mathbb{Z})$ be the inhomogeneous modular group with standard generators

$$
S=\left(\begin{array}{cc}
0 & -1 \\
1 & 0
\end{array}\right), T=\left(\begin{array}{ll}
1 & 1 \\
0 & 1
\end{array}\right) .
$$

We will be dealing with 2-dimensional irreducible representations $\rho: \Gamma \rightarrow G L(2, \mathbb{C})$ such that $\rho(T)$ is a diagonal unitary matrix,

$$
\rho(T)=\left(\begin{array}{cc}
e^{2 \pi i m_{1}} & 0 \\
0 & e^{2 \pi i m_{2}}
\end{array}\right) \quad\left(1>m_{1}>m_{2} \geq 0\right) .
$$

A holomorphic vector-valued modular form of integral weight $k$ associated to $\rho$ consists of the following data:

(i) a pair of holomorphic functions $f_{i}(\tau), i=1,2$, defined on the complex upper half-plane $\mathfrak{H}$ with $q$-expansions

$$
f_{i}(\tau)=\sum_{n \geq 0} a_{n, i} q^{m_{i}+n}
$$

(ii) functional equations

$$
\left.F\right|_{k} \gamma(\tau)=\rho(\gamma) F(\tau) \quad(\gamma \in \Gamma) .
$$

Here, $F(\tau)=\left(f_{1}(\tau), f_{2}(\tau)\right)^{t}$ i. 1 the column vector whose components are the functions $f_{i}(\tau)$ and $\left.\right|_{k}$ is the usual stroke operator in weight $k$ applied to each component. The coefficients $a_{n, i}$ in (1.2) are the Fourier coefficients of $f_{i}(\tau)$, or $F(\tau)$. The set

Received by the editors September 3, 2010 and, in revised form, February 8, 2011.

2010 Mathematics Subject Classification. Primary 11F99.

Supported by NSA and NSF.

${ }^{1}$ Superscript $t$ means transpose. 
$\mathcal{H}(k, \rho)$ of all holomorphic vector-valued modular forms of weight $k$ associated to $\rho$ is a finite-dimensional $\mathbb{C}$-linear space $(\underline{\mathrm{KM}}])$.

We say that $\rho$ is modular of level $N$ if $\operatorname{ker} \rho$ is a congruence subgroup of level $N$, i.e. $\operatorname{ker} \rho \supseteq \Gamma(N)$. In this case, the component functions of a vector-valued modular form $F(\tau) \in \mathcal{H}(k, \rho)$ are classical holomorphic modular forms of weight $k$ and level $N$. For the purposes of the present paper, the projective level of $\rho$ is a more useful invariant. If $\bar{\rho}$ is the projective representation of $\Gamma$ defined by the composition

$$
\Gamma \stackrel{\rho}{\rightarrow} G L(2, \mathbb{C}) \stackrel{\pi}{\rightarrow} P G L(2, \mathbb{C})
$$

( $\pi$ is canonical projection), we define the projective level $M$ of $\rho$ to be the order of $\bar{\rho}(T) \in P G L(2, \mathbb{C})$. We emphasize that we are not assuming that $\operatorname{ker} \bar{\rho}$ is a congruence subgroup.

There are infinitely many equivalence classes of 2-dimensional irreducible $\rho$, but it turns out ( $\mathrm{M} 2]$ ) that just 54 of them are modular. The ordinary level $N$ in these cases is some divisor of 120 , although not all $\rho$ of level dividing 120 are modular. On the other hand, it can be deduced from the tables in M2 (cf. Theorem 3.2 below) that

$$
\rho \text { is modular } \Leftrightarrow M \leq 5 .
$$

(1.5) is an algebraic characterization of those $\rho$ which are modular. The present paper is concerned with the problem of characterizing the modular $\rho$ by means of arithmetic properties of the Fourier coefficients of associated vector-valued modular forms. We are mainly interested in the space $\mathcal{H}(k, \rho)_{\mathbb{Q}} \subseteq \mathcal{H}(k, \rho)$ consisting of the $F(\tau)$ whose Fourier coefficients lie in $\mathbb{Q}$. As we will see (Lemma 4.2), $\mathcal{H}(k, \rho)_{\mathbb{Q}}$ is a $\mathbb{Q}$-form for $\mathcal{H}(k, \rho)$ (i.e. $\left.\mathcal{H}(k, \rho)=\mathbb{C} \otimes_{\mathbb{Q}} \mathcal{H}(k, \rho)_{\mathbb{Q}}\right)$ if the projective level of $\rho$ is finite.

Suppose that $F(\tau) \in \mathcal{H}(k, \rho)_{\mathbb{Q}}$ has component functions (1.2). We say that $F(\tau)$ has bounded denominators if there is a nonzero integer $B$ such that $B a_{n, i} \in \mathbb{Z}(n \geq$ $0, i=1,2)$. Otherwise, $F(\tau)$ has unbounded denominators. We can now state the

Conjecture. Suppose that $\rho$ has finite projective level. Then $\rho$ is modular if, and only if, there is some nonzero $F(\tau) \in \mathcal{H}(k, \rho)_{\mathbb{Q}}$ which has bounded denominators.

We make several remarks. The irreducibility of $\rho$ is implicitly assumed in the conjecture. If $\rho$ is modular, then, as we have explained, the components of a vector-valued modular form in $\mathcal{H}(k, \rho)$ are ordinary modular forms, in which case the bounded denominator property of Fourier coefficients is well known. So the conjecture really concerns the implication bounded denominators $\Rightarrow \rho$ modular. It is one of a hierarchy of similar conjectures about the modularity of vector-valued modular forms (of arbitrary finite dimension) whose Fourier coefficents are algebraic with bounded denominators. Other special cases that have been discussed in the literature include modular forms on noncongruence subgroups (cf. AtkinSwinnerton-Dyer AS and Kurth-Long [KL1, KL2]), and generalized modular forms (Kohnen-Mason ( KoM1, [KoM2]).

The main result of the present paper is the following:

Theorem 1. Suppose that $\rho$ has finite projective level $M$, and that $M$ does not divide 60. Then the components of every nonzero vector-valued modular form $F(\tau) \in \mathcal{H}(k, \rho)_{\mathbb{Q}}$ have unbounded denominators. 
By Theorem 1, a counterexample to the conjecture necessarily has projective level $M \mid 60$. There are approximately 350 equivalence classes of $\rho$ satisfying this condition, including of course the 54 classes which are modular. In particular, Theorem 1 proves the conjecture for all but finitely many equivalence classes of $\rho$.

The proof of Theorem 1 depends on results in [M1] and [M2] (see also [MM]) describing $\mathcal{H}(\rho)=\bigoplus_{k} \mathcal{H}(k, \rho)$ as a module over a certain ring $\mathcal{R}$ of differential operators. There is a minimal weight $k_{0}$ for which $\mathcal{H}\left(k_{0}, \rho\right)$ is nonzero, and this space is 1-dimensional with basis $F_{0}(\tau)$, say. $F_{0}(\tau)$ generates $\mathcal{H}(\rho)$ considered as an $\mathcal{R}$-module, and the general idea of the proof is to reduce questions about arbitrary $F(\tau)$ to questions about $F_{0}(\tau)$. The components of $F_{0}(\tau)$ span the solution space of a certain modular linear differential equation ([M1]) which has $q=0$ as a regular-singular point, and the Fuchsian theory provides a recursive formula for the corresponding Fourier coefficients. Assuming (as we may) that $F_{0}(\tau)$ has rational Fourier coefficients and that $M$ does not divide 60, we can exploit the recursion to obtain (Proposition 4.1) the exact power of $p$ dividing the denominators of the Fourier coefficients $a_{n, i}$ of $F_{0}(\tau)$ whenever $p$ is a prime dividing $M /(M, 60)$. The power of $p$ is strictly increasing for $n \rightarrow \infty$, and in particular $F_{0}(\tau)$ satisfies the unbounded denominator property. Together with the structure of $\mathcal{H}(\rho)$ as an $\mathcal{R}$ module, this result can then be used to deduce Theorem 1 for general $F(\tau)$.

The paper is organized as follows. In Section 2 we cover the background needed from [M1, M2]. In Section 3 we discuss the projective level $M$ and related invariants, and in particular we give (Lemma 3.2) a direct proof of (1.5) which does not rely on the tables in $\mathrm{M} 2$. Section 4 contains the proof of Theorem 1, and Section 5 contains some concluding remarks.

\section{BACKGROUND}

We review the notation and results we will need from [M1], [M2]. The $\mathbb{Z}$-graded algebra of holomorphic modular forms on $\Gamma$ is

$$
\mathcal{M}=\bigoplus_{k \geq 0} \mathcal{M}_{k}=\mathbb{C}\left[E_{4}(\tau), E_{6}(\tau)\right]
$$

where $E_{2 k}(\tau)=1-\frac{2 k}{B_{k}} \sum_{n \geq 1} \sigma_{2 k-1}(n) q^{n}$ is the usual normalized Eisenstein series of weight $2 k \geq 4$. The modular derivative in weight $k$ is the operator

$$
D_{k}=q \frac{d}{d q}+k E_{2}
$$

with $E_{2}(\tau)=-\frac{1}{12}+2 \sum_{n \geq 1} \sigma_{1}(n) q^{n} . \quad D_{k}: \mathcal{M}_{k} \rightarrow \mathcal{M}_{k+2}$ defines a degree 2 derivation $D: \mathcal{M} \rightarrow \mathcal{M}$ whose restriction to $\mathcal{M}_{k}$ is $D_{k}$. We often write $D$ in place of $D_{k} . \mathcal{M}$ and $D$ generate the ring $\mathcal{R}$ of skew polynomials whose elements are (noncommutative) polynomials $\sum_{i} m_{i} d^{i}, m_{i} \in \mathcal{M}$, satisfying the relation $d m-$ $m d=D(m)$.

Let the assumptions and notation be as in Section 1. The ring of holomorphic vector-valued modular forms with respect to $\rho$ is a $\mathbb{Z}$-graded linear space

$$
\mathcal{H}(\rho)=\bigoplus_{k=0}^{\infty} \mathcal{H}\left(k_{0}+2 k, \rho\right)
$$

where $k_{0}$ is the least weight for which a nonzero form exists. Then

$$
k_{0}=6\left(m_{1}+m_{2}\right)-1 \text {, }
$$


and $\mathcal{H}\left(k_{0}, \rho\right)=\mathbb{C} F_{0}$ is 1-dimensional. Let $f_{1}(\tau), f_{2}(\tau)$ be the component functions of $F_{0}(\tau)$ with Fourier coefficients $a_{n, i}$. The leading coefficients $a_{0, i}$ are nonzero and we may, and shall, assume that $a_{0, i}=1, i=1,2$. There is a natural componentwise action of $\mathcal{R}$ on elements in $\mathcal{H}(\rho)$ which turns the latter space into a $\mathbb{Z}$-graded left $\mathcal{R}$-module. Indeed, $\mathcal{H}(\rho)=\mathcal{R} F_{0}$ is a cyclic $\mathcal{R}$-module with generator $F_{0}$. Moreover $\mathcal{H}(\rho)$ is a free $\mathcal{M}$-module with free generators $F_{0}, D F_{0}$.

$f_{1}(\tau)$ and $f_{2}(\tau)$ form a fundamental system of solutions of a modular linear differential equation (MLDE) of weight $k_{0}$ and order 2; namely,

$$
D_{k_{0}}^{2} f+\kappa_{1} E_{4} f=0
$$

where

$$
\kappa_{1}=\left(1-36\left(m_{1}-m_{2}\right)^{2}\right) / 144
$$

and $D_{k_{0}}^{2}=D_{k_{0}+2} \circ D_{k_{0}}$. The MLDE (2.1) can be rewritten as

$$
q^{2} \frac{d^{2} f}{d q^{2}}+\left[1+2\left(k_{0}+1\right) E_{2}\right] q \frac{d f}{d q}+\left[k_{0}\left(k_{0}+1\right) E_{2}^{2}+\left(\kappa_{1}+k_{0} / 144\right) E_{4}\right] f=0,
$$

making it clear that $q=0$ is a regular singular point.

There is a recursive formula for the Fourier coefficients $a_{n, i}$ (e.g. [ $[\mathrm{H}$, p. 157 et seq., especially display (5.3.13)). We review the details as we will need them later. Set

$$
\begin{aligned}
\sum_{n=0}^{\infty} u_{n} q^{n} & =1+2\left(k_{0}+1\right) E_{2}(q)=\left(1-m_{1}-m_{2}\right)+O(q), \\
\sum_{n=0}^{\infty} v_{n} q^{n} & =k_{0}\left(k_{0}+1\right) E_{2}(q)^{2}+\left(\kappa_{1}+k_{0} / 144\right) E_{4}(q)=m_{1} m_{2}+O(q), \\
I_{0}(s) & =s^{2}-\left(m_{1}+m_{2}\right) s+m_{1} m_{2}, \\
I_{j}(s) & =u_{j} s+v_{j}, j \geq 1 .
\end{aligned}
$$

Explicitly,

$$
\begin{aligned}
u_{n}= & 24\left(m_{1}+m_{2}\right) \sigma_{1}(n), n \geq 1, \\
v_{n}= & k_{0}\left(m_{1}+m_{2}\right)\left(24 \sum_{r=1}^{n-1} \sigma_{1}(r) \sigma_{1}(n-r)-2 \sigma_{1}(n)\right) \\
& +10\left(m_{1}+m_{2}-6\left(m_{1}-m_{2}\right)^{2}\right) \sigma_{3}(n), n \geq 1, \\
I_{0}\left(n+m_{1}\right)= & n\left(n+m_{1}-m_{2}\right), \\
I_{j}\left(n+m_{1}-j\right)= & \left(n+m_{1}-j\right) u_{j}+v_{j}, j \geq 1 .
\end{aligned}
$$

The recursive formula is then given (setting $a_{n}=a_{n, 1}$ ) by $a_{0}=1$ and

$$
a_{n}=-\sum_{j=1}^{n} a_{n-j} \frac{I_{j}\left(m_{1}+n-j\right)}{I_{0}\left(m_{1}+n\right)}, \quad n \geq 1
$$

There is an analogous formula for the coefficients of $f_{2}(\tau)$.

\section{The PRojective LeVel $M$}

We retain the notation of the previous subsection.

Lemma 3.1. Suppose that $\rho$ has finite projective level $M$. Then $m_{1}, m_{2}$ and all Fourier coefficients $a_{n, i}$ lie in $\mathbb{Q}$. 
Proof. The assumption of the lemma is that $\bar{\rho}(T)$ has finite order $M$. Then (1.1) shows that $m_{1}, m_{2} \in \mathbb{Q}$. It then follows inductively using (2.2) and (2.3) that each Fourier coefficient is also rational.

From now on we will always assume that the projective level is finite. Introduce integers $a, b, c, d, N$ as follows:

$$
\begin{aligned}
& m_{1}=a / N, m_{2}=b / N,(a, b, N)=1, N>a>b \geq 0, \\
& c=(a-b, N), a-b=c d, N=c M .
\end{aligned}
$$

$N$ is the order of the matrix $\rho(T)$ (considered as an element of the group $\rho(\Gamma)$ ), which we also call the level of $\rho$. Let

$$
\Delta(N)=\left\langle\gamma T^{N} \gamma^{-1} \mid \gamma \in \Gamma\right\rangle
$$

be the normal closure of $T^{N}$ in $\Gamma$. Thus $\Delta(N) \subseteq \operatorname{ker} \rho$ and $\operatorname{ker} \rho$ has level $N$ in the sense of [W]. $M$ is the projective level of $\rho$. The following result includes (1.5).

Lemma 3.2. The following are equivalent:

(a) $\rho(\Gamma)$ is finite.

(b) $\bar{\rho}(\Gamma)$ is finite.

(c) $\rho$ has projective level $M \leq 5$.

(d) $\rho$ is modular of level $N$.

Proof. An old theorem of Schur $\left[\underline{S}\right.$ says that the commutator subgroup $G^{\prime}$ is a finite subgroup of a group $G$ if the index $|G: Z(G)|$ of the center is finite. Because $\left|\Gamma: \Gamma^{\prime}\right|$ is finite, it follows that $\rho(\Gamma)$ is finite if $|\rho(\Gamma): Z(\rho(\Gamma))|$ is finite. Since $\rho(\Gamma)$ is an irreducible group of linear transformations, we have $Z(\rho(\Gamma))=\rho(\Gamma) \cap Z$, where we have set $Z=Z(G L(2, \mathbb{C}))$. Since $\bar{\rho}(\Gamma)=\rho(\Gamma) Z / Z \cong \rho(\Gamma) / Z(\rho(\Gamma))$, the equivalence of (a) and (b) in Theorem 3.2 follows. The implication (a) $\Rightarrow$ (d) is proved in [M2], Theorem 3.5, while the converse is well known.

It is also well known (e.g. [W]) that if $M \leq 5$, then $\Delta(M)=\Gamma(M)$ is the principal congruence subgroup of $\Gamma$ of level $M$. Since $\Delta(M) \subseteq \operatorname{ker} \bar{\rho}$ it follows that if $M \leq 5$, then $\operatorname{ker} \bar{\rho}$ contains $\Gamma(M)$ and in particular has finite index in $\Gamma$. This establishes the implication $(\mathrm{c}) \Rightarrow(\mathrm{b})$.

Finally, we establish the implication (a) $\Rightarrow(\mathrm{c})$. Indeed, if (a) holds we may, and now shall, assume that $\rho$ is a unitary representation. In this case, $\bar{\rho}(\Gamma)$ is isomorphic to a subgroup of $P S U(2, \mathbb{C}) \cong S O(3, \mathbb{R})$. From Klein's classification of the finite subgroups of $S O(3, \mathbb{R})$, it follows that $\bar{\rho}(\Gamma)$ is one of the following: cyclic, dihedral, $A_{4}, S_{4}$, or $A_{5}$. The first case is excluded since $\rho$ is irreducible. In the last three cases, the elements of $\bar{\rho}(\Gamma)$ have order at most 5 , and in particular $M \leq 5$. In the dihedral case, $\bar{\rho}(T)$ generates the commutator quotient of $\bar{\rho}(\Gamma)$ since $T$ generates the commutator quotient of $\Gamma$, so that $M \leq 4$ in this case. We have thus established that (c) holds in all cases, and the proof of Lemma 3.2 is complete.

We record some numerical restrictions on the integers $a, b, c, d, M, N$.

Lemma 3.3. The following hold:
(a) $N \mid 6(a+b)$,
(b) $(c, M) \mid 2$,
(c) $4 \mid M \Rightarrow 2 \| c$,
(d) $c \mid(N, 12)$. 
Proof. Because $\rho$ is irreducible, $\rho\left(S^{2}\right)= \pm I_{2}$; in particular, $\operatorname{det} \rho\left(S^{2}\right)=1$. It follows from the relations in $\Gamma$ that $\operatorname{det} \rho\left(T^{6}\right)=1$, that is, $6\left(m_{1}+m_{2}\right) \in \mathbb{Z}$. Part (a) follows from this.

From (a) we obtain $c|6(a \pm b) \Rightarrow c|(12 a, 12 b, N) \Rightarrow c \mid(N, 12)$, establishing (d). If $3 \mid(c, M)$, then $3|a \pm b \Rightarrow 3|(a, b, N)=1$, a contradiction. This shows that $(c, M) \mid 4$. If $(c, M)=4$, then $16|N| 6(a+b) \Rightarrow 4|a \pm b \Rightarrow 2|(a, b, N)=1$, a contradiction which proves (b). Finally, $4|M \Rightarrow 2| a+b \Rightarrow 2 \mid a-b=c d$. As $(d, M)=1,2 \mid c$. This proves (c) and completes the proof of the lemma.

Lemma 3.4. $M \neq 6$.

Proof. Assume that $M=6$. Because $\rho$ is irreducible, it follows from [M2], Theorem 3.1, that $e^{2 \pi i(a-b) / N}=e^{2 \pi i d / 6}$ is not a primitive sixth root of unity. But then $(d, M)=(d, 6) \neq 1$, a contradiction.

Lemma 3.5. If $N=6,8$ or 12 , then $M \leq 5$ and $\rho$ is modular.

Proof. Suppose that $N=6$. We are done unless perhaps $M=6$, and this is not possible by Lemma 3.4. Now assume that $N=8$ or 12 . Since $N \mid 6(a+b)$, we have $2 \mid a+b$, whence $2 \mid a-b$ and $2 \mid c$. If $c=2$, then either $M=4$ as required or else $M=6$, contradicting Lemma 3.4. On the other hand, if $c \geq 3$, then $M \leq N / 3 \leq 4$. This completes the proof of the lemma.

\section{Proof of Theorem 1}

For a prime number $p$ define $\nu_{p}: \mathbb{Q}^{*} \rightarrow \mathbb{Z}$ as follows: for nonzero integers $m, n, \nu_{p}(n)=a$ if $n=p^{a} l$ with $(l, p)=1$, and $\nu_{p}(m / n)=\nu_{p}(m)-\nu_{p}(n)$. Set

$$
M=(M, 60) Q .
$$

Lemma 4.1. Let $p$ be a prime such that $p \mid Q$. Then the $n$th Fourier coefficient $a_{n}$ of $f_{1}(\tau)$ satisfies

$$
\nu_{p}\left(a_{n}\right)=-\nu_{p}\left(Q^{n} n !\right) .
$$

In particular, $\nu_{p}\left(a_{n}\right)$ is strictly decreasing for $n \geq 0$.

Proof. We prove the theorem by induction on $n$. The case $n=0$ holds because $a_{0}=1$. In the notation of Section 3, the recursion (2.3) reads as follows:

$$
\begin{aligned}
a_{n} & \left.=\frac{-1}{n c(M n+d)} \sum_{j=1}^{n} a_{n-j}\left\{(a+N(n-j)) u_{j}+N v_{j}\right)\right\} \\
& =\frac{-1}{n c(M n+d)} \sum_{j=1}^{n} a_{n-j} s_{j}(n),
\end{aligned}
$$

where we have set

$$
s_{j}(n)=(a+N(n-j)) u_{j}+N v_{j} .
$$


Then

$$
\begin{aligned}
s_{j}(n)= & \left\{\frac{2(a+b) \sigma_{1}(j)((12 n-12 j+1) N+6(a-b))}{N}\right\} \\
& +\frac{10 \sigma_{3}(j)\left((a+b) N-6(a-b)^{2}\right)}{N} \\
& +\frac{24(a+b)(6(a+b)-N)}{N} \sum_{r=1}^{j-1} \sigma_{1}(r) \sigma_{1}(j-r) \\
\equiv & -\frac{60 \sigma_{3}(j) c d^{2}}{M}(\bmod 2 \mathbb{Z}),
\end{aligned}
$$

where we used Lemma 3.3(a) for the last congruence. Using Lemma 3.3(b), we deduce from (4.3) that

$$
\nu_{p}\left(\frac{s_{j}(n)}{c}\right) \geq-\nu_{p}(Q)
$$

with equality if $j=1$.

Let

$$
b_{n, j}=\frac{a_{n-j}}{n} \frac{1}{M n+d} \frac{s_{j}(n)}{c}
$$

denote the $j$ th term on the right-hand side of (4.2). Using induction, (4.4) and $(M, d)=1$, we have for $1 \leq j \leq n$ that

$$
\nu_{p}\left(b_{n, j}\right) \geq-\nu_{p}\left(Q^{n-j}(n-j) !\right)-\nu_{p}(n)-\nu_{p}(Q) \geq-\nu_{p}\left(Q^{n} n !\right),
$$

and the two inequalities are both equalities if, and only if, $j=1$. By the nonArchimedean property of $p$-adic valuations, it follows that

$$
\nu_{p}\left(a_{n}\right)=\nu_{p}\left(\sum_{j=1}^{n} b_{n, j}\right)=\nu_{p}\left(b_{n, 1}\right)=-\nu_{p}\left(Q^{n} n !\right) .
$$

This completes the proof of the lemma.

Let $\mathcal{M}_{\mathbb{Q}}$ be the space of (classical) holomorphic modular forms with rational Fourier coefficients. Similarly, $\mathcal{H}(\rho)_{\mathbb{Q}}$ and $\mathcal{H}(k, \rho)_{\mathbb{Q}}$ are the corresponding spaces of vector-valued modular forms whose components have rational Fourier coefficients. Then $\mathcal{H}(\rho)_{\mathbb{Q}}$ is a left $\mathcal{M}_{\mathbb{Q}^{-}}$-module, and $F_{0} \in \mathcal{H}\left(k_{0}, \rho\right)_{\mathbb{Q}}$ by Lemma 3.1

Lemma 4.2. The following hold:

$$
\begin{aligned}
& \text { (a) } \mathcal{H}(\rho)_{\mathbb{Q}}=\left\{\alpha F_{0}+\beta D F_{0} \mid \alpha, \beta \in \mathcal{M}_{\mathbb{Q}}\right\}, \\
& \text { (b) } \mathcal{H}(\rho)=\mathbb{C} \otimes_{\mathbb{Q}} \mathcal{H}(\rho)_{\mathbb{Q}} .
\end{aligned}
$$

Proof. Since $F_{0} \in \mathcal{H}(\rho)_{\mathbb{Q}}$ then also $D F_{0} \in \mathcal{H}(\rho)_{\mathbb{Q}}$, and we conclude that $\left\{\alpha F_{0}+\right.$ $\left.\beta D F_{0} \mid \alpha, \beta \in \mathcal{M}_{\mathbb{Q}}\right\} \subseteq \mathcal{H}(\rho)_{\mathbb{Q}}$. Now $\mathcal{M}=\mathbb{C} \otimes_{\mathbb{Q}} \mathcal{M}_{\mathbb{Q}}$, and the equality $\mathcal{H}(\rho)=$ $\mathcal{M} F_{0} \oplus \mathcal{M D F} F_{0}$ is proved in [M2] (cf. the discussion on p. 406 of [M2]). Part (b) follows.

Now let $G \in \mathcal{H}(\rho)_{\mathbb{Q}}$. Because $F_{0}, D F_{0}$ are free generators of $\mathcal{H}(\rho)$ considered as $\mathcal{M}$-modules, there are unique forms $\alpha, \beta \in \mathcal{M}$ with the property that $\alpha F_{0}+\beta D F_{0}=$ $G$. To complete the proof of part (a) we have to show that $\alpha, \beta \in \mathcal{M}_{\mathbb{Q}}$. 
Let $g_{i}=\sum_{n>0} b_{n, i} q^{n+m_{i}}, i=1,2$, be the components of $D F_{0}$. Thus, each $a_{n, i}, b_{n, i} \in \mathbb{Q}$. Let $\alpha=\sum_{n \geq 0} c_{n} q^{n}, \beta=\sum_{n \geq 0} d_{n} q^{n}$. We prove by induction on $n$ that each $c_{n}, d_{n} \in \mathbb{Q}$.

The leading column of $F_{0}$ is $\left(q^{m_{1}}, q^{m_{2}}\right)^{t}$, while that for $D F_{0}$ is $\left(\left(m_{1}-k_{0} / 12\right) q^{m_{1}}\right.$, $\left.\left(m_{2}-k_{0} / 12\right) q^{m_{2}}\right)^{t}$. Thus the $n$th coefficient column for $G$ is equal to

$$
c_{n}\left(\begin{array}{c}
1 \\
1
\end{array}\right)+\sum_{j=1}^{n} c_{n-j}\left(\begin{array}{c}
a_{j, 1} \\
a_{j, 2}
\end{array}\right)+d_{n}\left(\begin{array}{l}
m_{1}-k_{0} / 12 \\
m_{2}-k_{0} / 12
\end{array}\right)+\sum_{j=1}^{n} d_{n-j}\left(\begin{array}{c}
b_{j, 1} \\
b_{j, 2}
\end{array}\right)
$$

and by hypothesis this has rational entries. If $n=0$ this says that

$$
c_{0}\left(\begin{array}{c}
1 \\
1
\end{array}\right)+d_{0}\left(\begin{array}{l}
m_{1}-k_{0} / 12 \\
m_{2}-k_{0} / 12
\end{array}\right)
$$

is rational. We can write this in the form

$$
\left(\begin{array}{ll}
1 & m_{1}-k_{0} / 12 \\
1 & m_{2}-k_{0} / 12
\end{array}\right)\left(\begin{array}{c}
c_{0} \\
d_{0}
\end{array}\right) \in \mathbb{Q}^{2} .
$$

Since $m_{1} \neq m_{2}$ the matrix in the last display is invertible, so $c_{0}, d_{0} \in \mathbb{Q}$. This begins the induction. The inductive step follows by the same argument, using (4.6). This completes the proof of the lemma.

For a prime $p$, let $\mathcal{B}_{p} \subseteq \mathcal{H}(\rho)_{\mathbb{Q}}$ be the span of those vector-valued modular forms with the property that there is an integer $B$ such that $B F(\tau)$ has $p$-integral Fourier coefficients. It is well known that the Fourier coefficients of every element in $\mathcal{M}_{\mathbb{Q}}$ have this property. Consequently, $\mathcal{B}_{p}$ is an $\mathcal{M}_{\mathbb{Q}}$-submodule of $\mathcal{H}(\rho)_{\mathbb{Q}}$.

Lemma 4.3. Suppose that $p \mid Q$. Then $\mathcal{B}_{p}=\{0\}$.

Proof. We assume that $\mathcal{B}_{p} \neq\{0\}$ and derive a contradiction. We first prove that

$$
\mathcal{M}_{\mathbb{Q}} F_{0} \cap \mathcal{B}_{p}=\{0\} .
$$

Because $\nu_{p}(Q) \geq 1$, we know that (4.1) holds. Suppose that $0 \neq \alpha \in \mathcal{M}_{\mathbb{Q}}$ with $\alpha=\sum_{n>0} b_{n} q^{n}$. There is an integer $N$ such that $\nu_{p}\left(b_{n}\right) \geq N$ for all $n$. Let $c_{n}$ be the $n$th Fourier coefficient of the first component of $\alpha F_{0}$, so that

$$
c_{n}=\sum_{j=0}^{n} b_{n-j} a_{j} .
$$

Assume to begin with that $b_{0} \neq 0$, and choose any integer $s>\nu_{p}\left(b_{0}\right)-N$. Using (4.1) we find that if $j<p^{s}$, then either $b_{p^{s}-j} a_{j}=0$ or else

$$
\begin{aligned}
& \nu_{p}\left(b_{0} a_{p^{s}}\right)-\nu_{p}\left(b_{p^{s}-j} a_{j}\right) \\
& \quad=\nu_{p}\left(b_{0}\right)-p^{s} \nu_{p}(Q)-\nu_{p}\left(p^{s} !\right)-\nu_{p}\left(b_{p^{s}-j}\right)+j \nu_{p}(Q)+\nu_{p}(j !) \\
& \quad \leq \nu_{p}\left(b_{0}\right)-\nu_{p}(Q)\left(p^{s}-j\right)-s-N \\
& \quad<\nu_{p}\left(b_{0}\right)-s-N<0 .
\end{aligned}
$$

Now (4.8) and the non-Archimedean property of $\nu_{p}$ show that

$$
\begin{aligned}
\nu_{p}\left(c_{p^{s}}\right)=\nu_{p}\left(b_{0} a_{p^{s}}\right) & =\nu_{p}\left(b_{0}\right)-p^{s} \nu_{p}(Q)-\nu_{p}\left(p^{s} !\right) \\
& \leq \nu_{p}\left(b_{0}\right)-p^{s}-s .
\end{aligned}
$$

Therefore $\alpha F_{0}$ does not have bounded $p$-power. If $b_{0}=0$ we obtain a similar result by using the first nonvanishing coefficient of $\alpha$ in place of $b_{0}$ in the previous argument. This completes the proof of (4.7). 
Now let $0 \neq F \in \mathcal{B}_{p}$ have weight $k$. By Lemma 4.2(a) we have

$$
F=\alpha F_{0}+\beta D F_{0}
$$

with $\alpha, \beta \in \mathcal{M}_{\mathbb{Q}}$. Note that $\beta \neq 0$ by (4.7).

Apply $D$ to (4.9) to get

$$
(D \alpha) F_{0}+\alpha D F_{0}+(D \beta) D F_{0}+\beta D^{2} F_{0}=D F,
$$

and then use (2.1) to obtain

$$
(D \alpha) F_{0}+\alpha D F_{0}+(D \beta) D F_{0}-\kappa_{1} \beta E_{4} F_{0}=D F .
$$

Now use (4.9) and (4.10) to eliminate the $D F_{0}$ terms. We obtain

$$
\left((D \alpha) \beta-\kappa_{1} \beta^{2} E_{4}-\alpha^{2}-\alpha D \beta\right) F_{0}=\beta D F-(\alpha+D \beta) F .
$$

The coefficient of $F_{0}$ on the left-hand side of (4.11) lies in $\mathcal{M}_{\mathbb{Q}}$, while the right-hand side lies in $\mathcal{B}_{p}$. Thanks to (4.7), the only way this can happen is for both sides to be identically zero. Then the components of $F$ satisfy the first-order differential equation

$$
\beta D F-(\alpha+D \beta) F=0,
$$

and since they are linearly independent this is not possible. This completes the proof of Lemma 4.3, and with it that of Theorem 1 as well.

It follows easily from Lemma 3.3 that if $M \mid 60$, then $N \mid 120$. So the version of the main theorem stated in the abstract is indeed a special case of Theorem 1.

\section{Concluding Remarks}

Suppose that $\rho$ is not modular, and that $F(\tau) \in \mathcal{H}(k, \rho)_{\mathbb{Q}}$ is a nonzero vectorvalued modular form with bounded denominators. From Theorem 1 and Lemmas 3.4 and 3.5 it follows that $M|60, N| 120$. Indeed, there are only 13 possible pairs $(M, N)$, namely:

$$
\begin{aligned}
& (10,10),(10,20),(10,30),(10,60),(12,24),(15,15),(15,30), \\
& (15,60),(20,40),(20,120),(30,30),(30,60),(60,120) .
\end{aligned}
$$

It is straightforward, though unenlightening, to enumerate all of the equivalence classes of $\rho$ corresponding to these values using Theorem 3.1 of [M2].

The recursive formula (2.3) is convenient for machine calculation. Inspection of the denominators of the first thousand or so coefficients of the examples corresponding to the pairs $(M, N)$ in (5.1) shows that they steadily increase, with more and more primes occurring in the denominators. There seems to be no question that the conjecture is true! On the other hand, it is unclear whether there are primes $p$ (analogous to those dividing $Q$ in Lemma 4.1) which divide the denominators of the Fourier coefficients of $f_{1}$ to a power which is unbounded for $n \rightarrow \infty$. It is also unclear whether one should expect that all but a finite number of primes occur in the denominators of these Fourier coefficients. This property holds for certain classes of generalized modular forms ( KoM1, KoM2]) and is something that one expects to be a rather general feature of vector-valued modular forms. The arithmetic nature of the denominators of the Fourier coefficients of the components of $F_{0}$, whether $M$ divides 60 or not, appears to be quite interesting. 


\section{REFERENCES}

[AS] Atkin, O., and Swinnerton-Dyer, P., Modular forms on noncongruence subgroups, Proc. Symp. Pure Math., Vol. XIX, Amer. Math. Soc., Providence, R.I. (1971), 1-25. MR0337781 (49:2550)

[H] Hille, E., Ordinary Differential Equations in the Complex Domain, Dover Publications, New York, 1976. MR0499382 (58:17266)

[KM] Knopp, M., and Mason, G., On vector-valued modular forms and their Fourier coefficients, Acta Arithmetica 110, No. 2 (2003), 117-125. MR2008079 (2004j:11043)

[KoM1] Kohnen, W., and Mason, G., On generalized modular forms and their applications, Nagoya J. Math. 192 (2008), 119-136. MR2477614(2009m:11060)

[KoM2] Kohnen, W., and Mason, G., On the canonical decomposition of a generalized modular form, to appear in Proc. Amer. Math. Soc. (arXiv:1003.2407).

[KL1] Kurth, C., and Long, L., On modular forms for some noncongruence subgroups of $S L_{2}(\mathbb{Z})$, J. Numb. Th. 128, No. 7 (2008), 1989-2009. MR2423745 (2010c:11055)

[KL2] Kurth, C., and Long, L., On modular forms for some noncongruence subgroups of $S L_{2}(\mathbb{Z})$, II, Bull. Lond. Math. Soc. 41, No. 4 (2009), 589-598. MR2521354 (2010i:11053)

[MM] Marks, C., and Mason, G., Structure of the module of vector-valued modular forms, J. Lond. Math. Soc. 82, Part 1 (2010), 32-48. MR.2669639

[M1] Mason, G., Vector-valued modular forms and linear differential equations, Int. J. Numb. Th. 3, No. 3 (2007), 1-14. MR2352826 (2008h:11038)

[M2] Mason, G., 2-dimensional vector-valued modular forms, Ramanujan Journal 17 (2008), 405-427. MR2456842 (2009j:11070)

[S] Schur, I., Über die Darstellung der endlichen Gruppen durch gebrochene lineare Substitutionen, J. für die Reine und Ang. Math. 127 (1904), 20-50.

[W] Wohlfahrt, K., An extension of F. Klein's level concept, Ill. J. Math. 13 (1964), 529-535. MR0167533 (29:4805)

Department of Mathematics, University of California, Santa Cruz, Santa Cruz, CaliFORNIA 95064

E-mail address: gem@cats.ucsc.edu 